\title{
Effects of hydrocortisone-presensitized sugammadex on recovery from neuromuscular blockade induced by rocuronium: a rodent in vivo study
}

\section{Hey-Ran Choi ${ }^{1}$, Hong-Seuk Yang ${ }^{2}$, Jae-Moon Choi ${ }^{3}$, Chungon Park ${ }^{4}$, Junyong $\mathrm{In}^{5}$, and Yong Beom Kim ${ }^{4}$}

'Department of Anesthesiology and Pain Medicine, Seoul Paik Hospital, Inje University College of Medicine, Seoul, ${ }^{2}$ Department of Anesthesiology and Pain Medicine, Daejeon Eulji Medical Center, Eulji University College of Medicine, Daejeon, ${ }^{3}$ Department of Anesthesiology and Pain Medicine, Asan Medical Center, University of Ulsan College of Medicine, Seoul, ${ }^{4}$ Department of Anesthesiology and Pain Medicine, Gil Medical Center, Gachon University College of Medicine, Incheon, ${ }^{5}$ Department of Anesthesiology and Pain Medicine, Ilsan Hospital, Dongguk University College of Medicine, Goyang, Korea

Background: Sugammadex is a specific antagonist of aminosteroidal neuromuscular blocking agents with 1:1 binding to guest molecules. Sugammadex can also bind to other drugs having a steroid component in its chemical structure. In this in vivo experiment, we investigated the differences in the recovery of rocuronium-induced neuromuscular blockade using sugammadex pre-exposed with two different concentrations of hydrocortisone.

Methods: The sciatic nerves and tibialis anterior muscles of 30 adult Sprague-Dawley rats were prepared for the experiment. The sciatic nerves were stimulated using a train-of-four (TOF) pattern with indirect supramaximal stimulation at $20 \mathrm{~s}$ intervals. After $15 \mathrm{~min}$ of stabilization, a $250 \mu \mathrm{g}$ loading dose and $125 \mu \mathrm{g}$ booster doses of rocuronium were serially administered until > 95\% depression of the first twitch tension of TOF stimulation (T1) was confirmed. The study drugs were prepared by mixing sugamadex with the same volume of three different stock solutions ( $0.9 \%$ normal saline, $10 \mathrm{mg} / \mathrm{ml}$ hydrocortisone, and $100 \mathrm{mg} /$ $\mathrm{ml}$ hydrocortisone). The recovery of rats from neuromuscular blockade was monitored by assessing T1 and the TOF ratio (TOFR) simultaneously until T1 was recovered to $>95 \%$ and TOFR to $>0.9$.

Results: In the group injected with sugammadex premixed with a high concentration of hydrocortisone, statistically significant intergroup differences were observed in the recovery progression of T1 and TOFR $(\mathrm{P}<0.050)$.

Conclusions: When sugammadex was pre-exposed to a high dose of hydrocortisone only, recovery from neuromuscular blockade was delayed. Delayed recovery from neuromuscular blockade is not always plausible when sugammadex is pre-exposed to steroidal drugs.

Keywords: Hydrocortisone; Neuromuscular blockade; Neuromuscular blocking agent; Neuromuscular junction; Rocuronium; Sugammadex.

This is an Open Access article distributed under the terms of the Creative Commons Attribution Non-Commercial License (http://creativecommons.org/licenses/by-nc/4.0) which permits unrestricted non-commercial use, distribution, and reproduction in any medium, provided the original work is properly cited.

Copyright (C) the Korean Society of Anesthesiologists, 2022 


\section{INTRODUCTION}

Sugammadex is a prime antagonist of aminosteroidal neuromuscular blocking agents (NMBAs), especially rocuronium [1-3]. It directly encapsulates and inactivates rocuronium at the molecular level in a 1:1 manner [4]. Before the introduction of sugammadex, the main method of antagonizing neuromuscular blockade was by administering anticholinesterase, to increase acetylcholine levels in neuromuscular junctions, thereby competing with rocuronium in binding to postsynaptic nicotinic acetylcholine receptors $[5,6]$. However, this indirect manner of antagonizing neuromuscular blockade has a ceiling effect in reversing rocuronium-induced neuromuscular block, giving rise to limitations and considerations, such as the depth of neuromuscular block at the time of reversal [7]. In contrast, the mechanism of sugammadex-induced recovery from neuromuscular blockade is through the encapsulation of host molecules and their inactivation [4]. In sugammadex-induced antagonism, the depth of neuromuscular block at the time of reversal is irrelevant, which means that increasing the dose of sugammadex enables rapid recovery from a deep or intense neuromuscular block $[1,3]$. However, besides rocuronium, the host-guest binding property of sugammadex includes other molecules that have a steroid component [8]. Neverthless, their affinity with sugammadex is not as strong as the affinity between rocuronium and sugammadex, and such molecules have minimal or no effects on sugammadex-induced recovery from rocuronium-induced neuromuscular blockade $[9,10]$. Zwiers et al. [8] reported that the affinity between sugammadex and steroidal NMBAs is strong, such that it is difficult for other molecules to displace their hostguest bonds. Choi et al. [9] reported that sugammadex-induced recovery from neuromuscular blockade was not affected by the clinical concentration of remifentanil, although the recovery may be delayed when an extremely high concentration of remifentanil is used. Choi et al. [10] reported that dexamethasone and hydrocortisone do not affect sugammadex-induced reversal of neuromuscular blockade. These observations were made when the drugs were in the form of free molecules. In other words, hydrocortisone and dexamethasone competed with rocuronium to simultaneously bind sugammadex. However, in clinical settings, sugammadex is usually administered through the intravenous (IV) line, which is a common route for the delivery of other drugs. If drugs with good affinity to sugammadex are present in the IV access line, sugammadex can bind to these drugs. Consequently, this might affect sugammadex-rocuronium binding in the bloodstream, which, in turn, might affect the sugammadex-induced recovery from the neuromuscular blockade. Therefore, we hypothesized that sugammadex-induced recovery from the neuromuscular blockade might be affected when sugammadex is premixed with molecules that have an affinity for it. In this in vivo experiment, we investigated the differences in the recovery of rocuronium-induced neuromuscular blockade between groups of anesthetized rats injected with sugammadex pre-exposed with two different concentrations of hydrocortisone or the same dose of sugammadex alone. We compared the $\mathrm{T} 1$ twitch tensions and train-of-four (TOF) ratios (TOFR) between the groups.

\section{MATERIALS AND METHODS}

The study protocol was approved by the Ethics Committee of the Laboratory of Animal Research, Asan Institute of Life Science (Seoul, Korea) on May 1, 2019 (no. 2019-13-083). All methods were performed in accordance with the Animal Research: Reporting of In Vivo Experiments (ARRIVE) guidelines and regulations. Thirty male Sprague-Dawley (SD) rats with an average age of 4-6 weeks were used in this study. The average body weight at the time of main experiment was $240.53 \pm 6.01 \mathrm{~g}$ (range 225-250 g). All animals were bred at a constant temperature of $22^{\circ} \mathrm{C}$ under a regular diurnal cycle, with food and water supplied ad libitum. During the experiment, the temperature of the rats was monitored using a rectal thermistor and maintained at 35.5$37^{\circ} \mathrm{C}$ with a heat lamp.

Each rat was anesthetized with an intraperitoneal injection of $0.5 \mathrm{mg} / \mathrm{kg}$ urethane (Sigma-Aldrich Korea, Korea). Adequate anesthetic levels were confirmed by pinching the hind limbs with a clamp. After confirming that there were no responses to clamp pinching, preparations for the experiment were started. First, a tracheostomy was performed and a Y-tube was placed. Intrajugular venous cutdown was performed and an IV catheter was inserted. The tendons of both the tibialis anterior muscles were exposed and cut at their insertion sites. A black silk string was tied to each tendon and a lasso was made to hook the Grass FT03 force transducer (Grass Technologies, USA). The sciatic nerves in both gluteal regions were exposed and fixed to platinum bipolar electrodes. The rat was then placed on a tray with both knees clamped and fixed to the frames of the tray. Platinum bipolar electrodes at both sciatic nerves were connected to a 
Grass S88 stimulator (Grass Technologies), and the lassos at the tendons of both tibialis anterior muscles were hooked to the force transducer. Four $2 \mathrm{~Hz}$ indirect stimulations (TOF stimulations) were supplied to the sciatic nerves every $15 \mathrm{~s}$. Supramaximal stimulation was set at $10 \%$ above the level of the current with which there was no further increase in the twitch tensions of both tibialis anterior muscles. A stabilization time of 15 min was allowed with mechanical ventilation with ambient air at $90-110$ breaths/min and a tidal volume of $6-8 \mathrm{ml} / \mathrm{kg}$, which was supplied with a rodent ventilator model 683 (Harvard Apparatus, USA). Experiments were conducted when $\mathrm{T} 1$ was consistent and maintained during resting time. The inclusion criteria were T1 twitch $\geq 200$ $\mathrm{mN}$, maintained for $\geq 15 \mathrm{~min}$. However, the experiments were stopped and excluded if the monitored muscle twitch tensions were $\leq 200 \mathrm{mN}$ or serially decreased to $0 \mathrm{mN}$. Data were excluded when the $\mathrm{T} 1$ twitch tension gradually decreased before the addition of the study drugs or when the maximum recovery of $\mathrm{T} 1$ twitch tension was $\leq 50 \mathrm{mN}$ within $10 \mathrm{~min}$.

In the initial set of experiments, the cumulative dose-response data of rocuronium (MSD Korea, Korea) were obtained with a loading dose of $250 \mu \mathrm{g}$ and booster doses of $125 \mu$ g repeatedly injected until > 95\% depression of $\mathrm{T} 1$ was observed. The total dose of rocuronium used at that point was defined as the effective dose $\left(\mathrm{ED}_{95}\right)$. The next booster dose injection was considered when the depression in muscle twitch tension depression was $\leq 3 \%$ or tended to increase compared with the previous twitch tension. The study drugs were prepared by mixing $1 \mathrm{mg} / \mathrm{kg}$ sugammadex (MSD Korea) with the same volume of $0.9 \%$ isotonic saline (control group), $10 \mathrm{mg} / \mathrm{ml}$ hydrocortisone (SGX + Low group), and $100 \mathrm{mg} / \mathrm{ml}$ hydrocortisone (SGX + High group). Hydrocortisone was purchased from Hanall Biopharma (Korea). After confirming that the responses to the TOF stimulations were 0 , the $\mathrm{ED}_{95}$ of rocuronium was injected. When the T1 responses reappeared, the allocated experimental drugs were injected, and the $\mathrm{T} 1$ twitch tensions and TOFR were serially monitored and recorded. The time interval from the injection of the study drugs to the point of $95 \%$ recovery of $\mathrm{T} 1$ twitch tension was recorded and compared between the groups. TOFRs were simultaneously obtained, while the recovery of $\mathrm{T} 1$ was monitored. The regression curves of these variables were compared between groups. Muscle twitches were sensed and changed through an electric signals using Grass FT03 force transducers, which were displayed and stored using the PowerLab 4/26 data acquisition system (AD
Instruments, Australia) and LabChart 7 software (AD Instruments, USA). In addition, deeply anesthetized SD rats were placed in the $\mathrm{CO}_{2}$ chamber or removed from mechanical ventilation after IV injection of rocuronium and intraperitoneal injection of urethane. The protocol of the main experiment is summarized in Fig. 1.

Data are expressed as mean \pm standard deviation. Statistical analysis was performed using SPSS version 13.0 software (SPSS Inc., USA). Rats were allocated to each group (control, SGX + Low, and SGX + High) using random numbers generated with the following equation in Microsoft Excel 2010 program (Microsoft Office; Microsoft Corporation, USA); $f_{x}$ $=\left(\operatorname{INT}\left(\operatorname{RAND}()^{*} 3\right)\right)+1$. For group blinding, the principal investigator prepared premixed, non-labeled study drugs during the stabilization time and cumulative dose-response study of rocuronium, and provided them to the researcher when all TOF responses had disappeared. Recovery data were plotted by fitting nonlinear regression curves to group data. We used the equations $y=100+\Omega(x-b)^{3}$ and $y=1+$ $\lambda(x-c)^{3}$ to describe $\mathrm{T} 1$ and TOFR recovery, respectively $\left(\mathrm{R}^{2}\right.$ $>0.8$ ). In these equations, $y$ represents $\mathrm{T} 1$ or TOFR, $x$ represents the time set from $5 \% \mathrm{~T} 1$ recovery (taken as the zero point) or injection of the study drug, $b$ and $c$ respectively represent the virtual time to $>95 \% \mathrm{~T} 1$ recovery, and $>0.9$ TOFR, while the slope of each regression curve is denoted by $\Omega$ and $\lambda$. Differences in continuous variables among the groups were analyzed using analysis of variance, followed by the Bonferroni method for multiple pairwise comparisons. The mean group values of $\Omega$ and $\lambda$ were compared using the Kruskal-Wallis test. Statistical significance was set at $\mathrm{P}<$ 0.05 . The primary objective of the present experiment was to determine the differences in recovery progression from rocuronium-induced neuromuscular blockade between groups by comparing slopes of the regression curves, which are represented as $\Omega$ and $\lambda$. The sample size of the present study was calculated following previous experiments $[11,12]$ and a pilot study based on the slope of recovery time $(\Omega)$ of $\mathrm{T} 1$ to reach $>95 \%$ of the initial T1. Although there are some issues with sample size estimation in in vivo neuromuscular studies, the calculation suggested that 10 samples per group would be sufficient at an $\alpha=0.05$, a power of 0.80 , and a dropout rate of $10 \%$.

\section{RESULTS}

No statistically significant differences in body weight, weight of tibialis anterior muscles, and rocuronium dose 


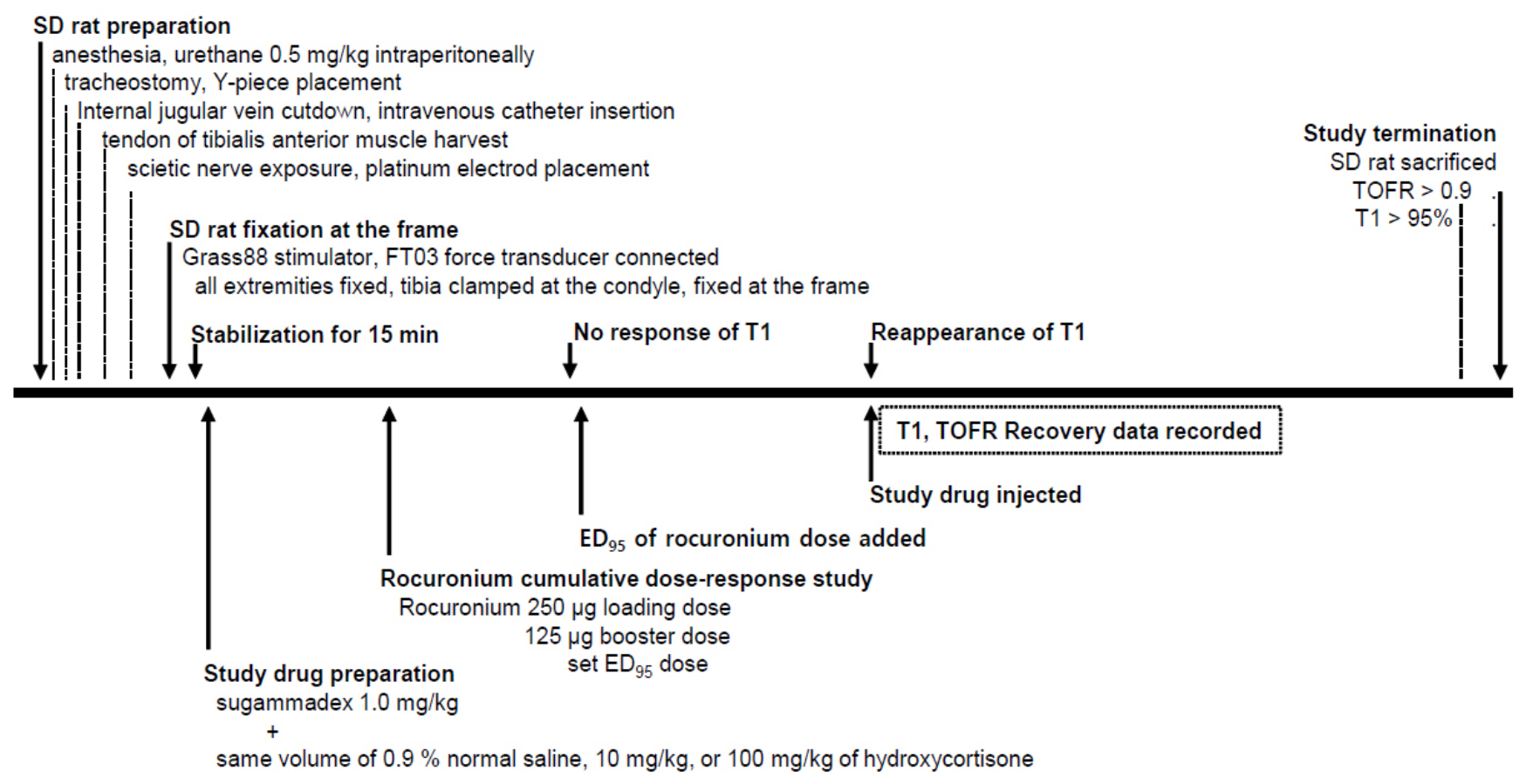

Fig. 1. Study protocol. SD: Sprague-Dawley, TOFR: train-of-four ratio, ED ${ }_{95}: 95 \%$ effective dose.

Table 1. Characteristics of Rats and Tissue Specimen

\begin{tabular}{|c|c|c|c|}
\hline Variable & Control $(n=10)$ & $\operatorname{SGX}+\operatorname{Low}(n=10)$ & SGX + High $(n=10)$ \\
\hline BW (g) & $240.48 \pm 6.34$ & $239.89 \pm 6.23$ & $241.24 \pm 5.45$ \\
\hline TA (mg) & $374.29 \pm 21.53$ & $375.72 \pm 23.72$ & $368.51 \pm 18.64$ \\
\hline $\operatorname{Roc}(\mu g)$ & $390.63 \pm 104.32$ & $375.06 \pm 102.60$ & $392.86 \pm 112.47$ \\
\hline
\end{tabular}

Data are expressed as mean \pm SD. BW: body weight of the rat, TA: wet weight of the tibialis anterior muscle, Roc: average dose of rocuronium, SGX: sugammadex. No statistically significant differences were observed between the groups $(P>0.05)$.

Table 2. Coefficients of Determination

\begin{tabular}{lccc}
\hline \multicolumn{1}{c}{ Variable } & Control $(n=10)$ & SGX + Low $(n=10)$ & SGX + High $(n=10)$ \\
\hline$R^{2}$ for T1 recovery & $0.96 \pm 0.01$ & $0.97 \pm 0.02$ & $0.94 \pm 0.05$ \\
$R^{2}$ for TOFR & $0.86 \pm 0.1$ & $0.87 \pm 0.08$ & $0.89 \pm 0.09$ \\
\hline
\end{tabular}

Data are expressed as mean \pm SD. The equation for the regression curves of T1 recovery was set as $y=100+\Omega(x-b)^{3}$. The equation for the regression curves of TOFR recovery was set as $y=1+\lambda(x-c)^{3}$. In these equations, $y$ represents T1 or TOFR, $x$ represents the time set from $5 \% \mathrm{~T} 1$ recovery (taken as the zero point) or injection of the study drug, $b$ and c represent virtual time for reaching $>95 \% \mathrm{~T} 1$ and $>0.9$ TOFR, respectively, and $\Omega$ and $\lambda$ represent the progression slope of each regression curve. SGX: sugammadex, TOFR: train-of-four ratio.

were observed among the groups (Table 1). Figs. 2 and 3 show the recovery data for $\mathrm{T} 1$ and TOFR, respectively. In Figs. 2A and 3A, the dot, triangle, and diamond symbols represent data obtained from the control, SGX + Low, and SGX + High groups, respectively. Solid, dashed, and dash-dot fitting lines represent the recovery progression and regression lines of the control, SGX + Low, and SGX + High groups, respectively. In Fig. 2, the regression line of each group is expressed as $y=100+\Omega(x-b)^{3}$, and the average values of $\Omega$ and $b$ were compared between the groups. The coefficeints of determination $\left(\mathrm{R}^{2}\right)$ of all groups were well over 0.7 as listed in Table 2. $\Omega$ in the SGX + High group was statistically different from that in the control or SGX + Low groups (Fig. 2B, $\mathrm{P}=0.001)$. The average $b$ represents the expected time to acnieve $100 \%$ recovery of the T1 twitch tension. In SGX + High, the average $b$ was $374.8 \mathrm{~s}$, which was significantly longer than that in the control (208.7 s) or SGX + Low (280.3 s) group $(\mathrm{P}<0.001$, and 0.037 , respectively). Fig. 3 shows the 


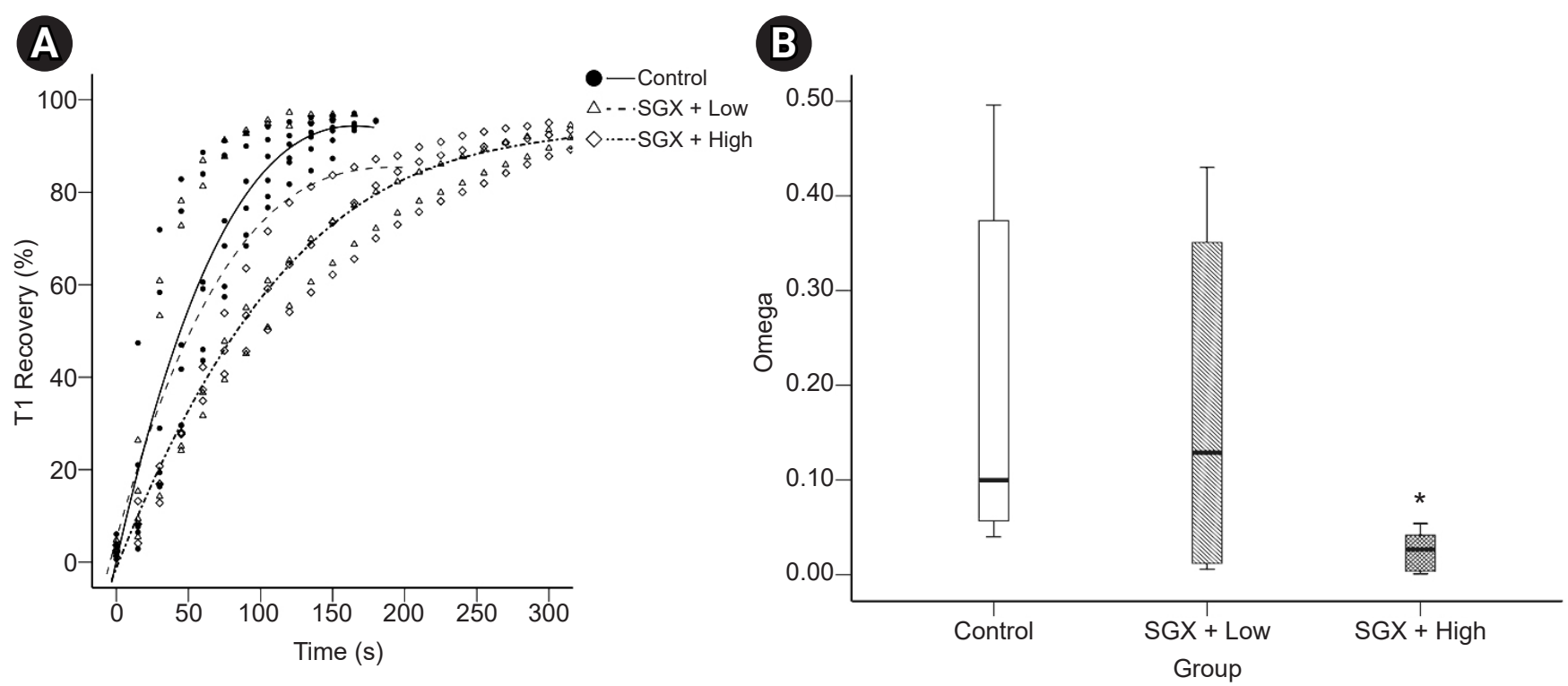

Fig. 2. Comparison of the progression of recovery of T1. We used the equation of $\mathrm{y}=100+\Omega(x-b)^{3}$ where $y$ represents T1, $x$ represents the time set from 5\% T1 recovery (taken as the zero point), $b$ represent the virtual time to $>95 \% \mathrm{~T} 1$ recovery, and $\Omega$ represent the slope of each regression curve. In (A), the T1 recovery progression in the SGX + High group (diamond, dashdot line) was significantly delayed comparing with the control (dot, solid line), or SGX + Low (triangle, dashed line) group. In the box plot in (B), the mean value of $\Omega$ in the SGX + High (cross hatched box) was significantly lower than that of the control (hollow box), or SGX + Low (single hatched box) groups ( ${ }^{\mathrm{P}}=$ 0.001). However, there were no significant differences in $\Omega$ between the control and SGX + Low groups $(P>0.05)$. T1: first twitch tension of train-of-four stimulation, SGX: sugammadex.
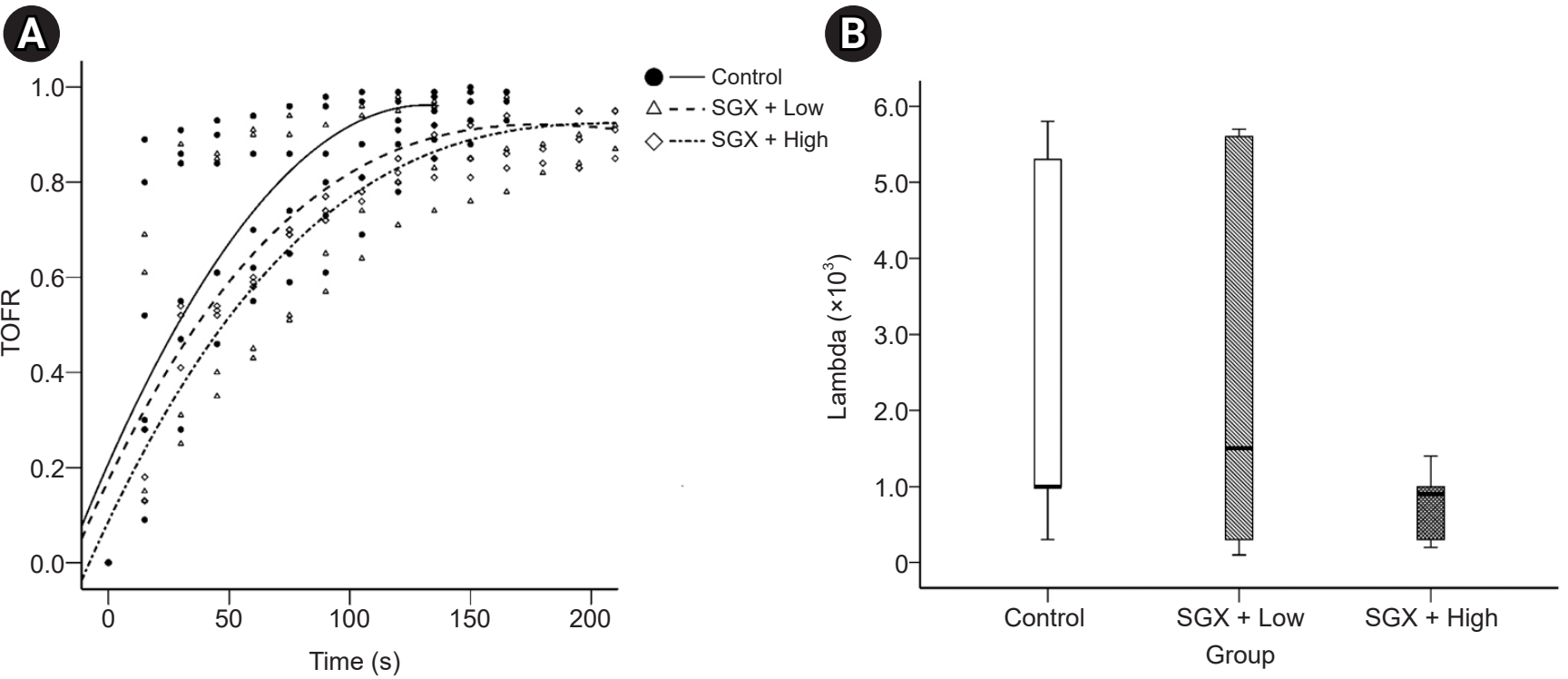

Fig. 3. Comparison of the progression of TOFR recovery. We used the equation of $y=1+\lambda(x-b)^{3}$ where $y$ represents TOFR, $x$ represents the time set from injection of the study drug (taken as the zero point), $c$ represent the virtual time to $>0.9$ TOFR, and $\lambda$ represent the slope of each regression curve. In (A), the TOFR in the SGX + Low (triangle, dashed line) and SGX + High (diamond, dash-dot line) groups showed significantly slower recovery than that of the control (dot, solid line) group. In the box plot in (B), the mean value of $\lambda$ in control (hollow box) group was significantly higher than that in the SGX + Low (single hatched box, P = 0.006) and SGX + High (cross hatched box, $\mathrm{P}=0.004)$ groups. T1: first twitch tension of train-of-four stimulation, SGX: sugammadex, TOFR: train-of-four ratio.

data for the recovery of TOFR, which appearsed to be delayed in the SGX + High group. The mean value of $\lambda$ in SGX + High group was lower than that in the control or SGX + Low groups (Fig. 3B, $\mathrm{P}=0.006$, and 0.004 , respectively). However, when comparing $\lambda$ between the control and SGX + Low groups, there were no statistically significant differences (P 
$=0.978)$. When comparing the expectation time to 1.0 of TOFR recovery, we did not find any statistically significant differences between groups $(\mathrm{P}=0.762,1.000$, and 0.127 between the control vs. SGX + Low, SGX + Low vs. SGX + High, and control vs. SGX + High groups, respectively).

\section{DISCUSSION}

This in vivo experiment demonstrated that $\mathrm{T} 1$ recovery might be delayed when sugammadex is pre-exposed with hydrocortisone before binding to rocuronium. In the present experiment, we premixed sugammadex with two different concentrations of hydrocortisone and injected the mixture into SD rat; thereafter, we simulated the environment in which sugammadex is bind to hydrocortisone before it binds to rocuronium. In clinical practice, IV extension lines and three-way connectors are often used because of the patient positioning and to allow easy access to additional fluids or drugs. The longer these lines are, the more time it takes for the drugs to remain inside them. Therefore, it is possible for the administered drugs to react with each other in the IV line before they reach the body to exert their own effects. Sugammadex binds to these molecules in a 1:1 manner and chelates them. However, this reaction often occurs when a steroid component is present in the structure of molecules. Zwiers et al. [8] reported that, although most drugs have a lower affinity to sugammadex than rocuronium and have minimal effects on sugammadex-induced recovery from neuromuscular blockade, some drugs have a fair affinity to sugammadex including drugs used during anesthesia induction and maintenance. Hydrocortisone is an adjuvant drug that has long been used during anesthesia and surgery.

Hydrocortisone and other glucocorticoids are used to treat reactive adverse events of the airway during intubation, acute nerve injury, nausea, and vomiting, as well as for immunosuppression during organ transplantation [13]. Recently, several recommendations have been published regarding the perioperative use of glucocorticoids $[14,15]$. Therefore, perioperative administration of glucocorticoids is increasingly being performed according to surgical and anesthetic conditions to improve prognosis, although more reliable evidence is still required [16]. Many researchers have investigated the potential for interactions between sugammadex and drugs other than rocuronium [17-19]. Generally, cyclodextrin interacts with benzene primarily through attractive van der Waals interactions, whose values change from approximately -10 to $-13 \mathrm{kcal} / \mathrm{mol}$ during the binding of the molecules, which is opposed to the loss of configurational entropy [20]. As the backbone of sugammadex is a $\gamma$-cyclodextrin molecule, it may follow a mechanism similar to that of "guest" molecules. Stronger binding forces make the host-guest species more strongly immobilized. This leads to greater losses in configurational entropy $[20,21]$. Consequently, sugammadex molecules that react with guest molecules rather than rocuronium molecules do not react like free molecules. In this situation, the patterns of recovery from rocuronium-induced neuromuscular blockade will not be the same as those with "free" sugammadex. Our study followed this concept and obtained favorable results. In the present study, sugammadex was pre-exposed to two different concentrations of hydrocortisone, $(10 \mathrm{mg} / \mathrm{ml}$ and 100 $\mathrm{mg} / \mathrm{ml}$ ), and the different mixtures showed different results. When $10 \mathrm{mg} / \mathrm{ml}$ hydrocortisone was used, the progression of the $\mathrm{Tl}$ recovery was not different from that of the control group. Tl and TOFR recovery was delayed in the group administered $100 \mathrm{mg} / \mathrm{ml}$ hydrocortisone. To find the differences between the groups, we compared $\Omega$ and $\lambda$ (representing the slope of each regression curve). Most of the data obtained eventually reached full recovery. These were not only the results of the action of sugammadex but also the results of spontaneous recovery from rocuronium-induced neuromuscular blockade. We focused on the pattern of recovery progression from the neuromuscular blockade. The values of $\Omega$ and $\lambda$ of SGX + High group were statistically different from those of the other groups (Figs. 2B, 3B). The association rate constant $\left(\mathrm{k}_{\text {ass }}\right)$ of hydrocortisone is $5.48 \times 10^{4} \mathrm{~mol} / \mathrm{L}$, which is much weaker than that of rocuronium $\left(1.79 \times 10^{7}\right.$ $\mathrm{mol} / \mathrm{L})$. This implies that the reaction between sugammadex and hydrocortisone might not be as rapid and strong as the reaction between sugammadex and rocuronium. Accordingly, more "free" sugammadex molecules might have remained in the group that was exposed to a low concentration of hydrocortisone. This explained that there were no significant differences between the control and SGX + Low groups. In the SGX + High group, there was a relatively large amount of hydrocortisone molecules, although the affinity of hydrocortisone to sugammadex was relatively weak, and the recovery of $\mathrm{T} 1$ and TOFR was delayed when a relatively low dose of sugammadex was used.

This study had several limitations. First, this was a rodent in vivo experiment. As we used SD rats for neuromuscular physiology experiments in our laboratory, the authors are familiar with its handling. In the clinical setting, the recovery time is approximately $\leq 5$ min depending on the dose of 
sugammadex used. The use of $2 \mathrm{mg} / \mathrm{kg}$ sugammadex during a moderate neuromuscular block and $4 \mathrm{mg} / \mathrm{kg}$ sugammadex during a deep neuromuscular block resulted in $\leq 3$ and 5 min of recovery time to TOFR $>0.9$, respectively $[3,22,23]$. However, when we designed the present study and performed a pilot study, the TOFR recovery time was $\leq 1 \mathrm{~min}$ when $2 \mathrm{mg} / \mathrm{kg}$ of sugammadex was used. Moreover, T1 was fully recovered within $30 \mathrm{~s}$. Within this time interval, sufficient cumulative data of $\mathrm{T} 1$ and TOFR per rat could not be obtained because only one or two data points were obtained. Therefore, we reduced dose of sugammadex to $1 \mathrm{mg} /$ $\mathrm{kg}$. This discrepancy between the in vivo experiment and the clinical setting means that the postoperative residual block remains an issue [24,25]. Second, this study focused on sugammadex premixed with hydrocortisone. In the pilot study, we needed to determine the adequate concentration of hydrocortisone because we found no data about the amount of hydrocortisone remaining in the IV lines after injection. Hydrocortisone stock solutions were prepared at 0.1 , 1,10 , and $100 \mathrm{mg} / \mathrm{ml}$ in the pilot study. When the 0.1 and 1 $\mathrm{mg} / \mathrm{ml}$ stock solutions were used, no statistical significance was observed. Meanwhile, stock solutions with concentrations of 10 and $100 \mathrm{mg} / \mathrm{ml}$ showed ambiguous results or statistical differences. Although the total volume of the IV extension line varies according to its length, we considered that it contained $8-10 \mathrm{ml}$ of fluid. As such, we speculated that the minimum concentration of hydrocortisone might be obtained when $100 \mathrm{mg} / \mathrm{ml}$ hydrocortisone was mixed with $10 \mathrm{ml}$ of fluid. Therefore, we selected 10 and $100 \mathrm{mg} / \mathrm{ml}$ stock solutions in the main experiment. We used a hydrocortisone product available at $100 \mathrm{mg} / \mathrm{vial}$. It is commonly melted in $1 \mathrm{ml}$ of solvent and injected via a bolus or mixed with IV fluid. Therefore, sugammadex is rarely pre-exposed with the same concentration of hydrocortisone that we used in the present experiment because it is diluted with the fluid in the IV line and sugammadex may not directly mix with the hydrocortisone fluid. We believe that there is a low possibility that sugammadex is completely pre-exposed by hydrocortisone. Although these drugs are administered simultaneously, the chemical and biophysiological environment in the clinical setting is different from that in our experiment. However, because there are products containing $500 \mathrm{mg}$ hydrocortisone per vial and extremely high doses of steroids can be used as steroid pulse therapy or for other purposes of the positive effects of steroids [16]. The environment we set in the present study might still occur in clinical practice and sugammadex-induced recovery from neuromuscular block- ade might be delayed by pre-exposure with steroids. Finally, the regression equations we used were valid only for the present experiment data. In the present study, data collection was ended as soon as T1 recovery or TOFR reached $100 \%$ or 0.9 , respectively. As we mentioned earlier, the present data fit well by these equations $\left(\mathrm{R}^{2}>0.8\right)$ within the range of time we monitored and collected the data. However, these results were confined to the present data because the data after recovery of $\mathrm{T} 1$ and TOFR were not included in this study. In the clinical setting, if initial calibration failed or acceleromyography was used for neuromuscular monitoring, T1 and TOFR might be over 100\% and 1.0, respectively. However, under general conditions, T1 and TOFR remained stable after they reached full recovery.

In conclusion, our experiment showed that although sugammadex has a greater affinity for rocuronium than most other drugs, it is possible that the $\mathrm{T} 1$ and TOFR recovery times might be delayed when sugammadex is pre-exposed to drugs with a relatively high affinity to it. Fortunately, delays in the recovery from neuromuscular blockade induced by sugammadex which is pre-exposed to steroids are not mandatory. However, sugammadex-induced recovery from neuromuscular blockade might be delayed when there is sufficient time and quantity of steroids pre-exposed to sugammadex.

\section{FUNDING}

None.

\section{CONFLICTS OF INTEREST}

Junyong In has been an editor of the Anesthesia and Pain Medicine since 2017; however, he was not involved in the peer reviewer selection, evaluation, or decision process of this article. No other potential conflicts of interest relevant to this article were reported.

Yong Beom Kim has been an editor of the Anesthesia and Pain Medicine since 2015; however, he was not involved in the peer reviewer selection, evaluation, or decision process of this article. No other potential conflicts of interest relevant to this article were reported.

\section{DATA AVAILABILITY STATEMENT}

The datasets generated during and/or analyzed during the 
current study are available from the corresponding author on reasonable request.

\section{AUTHOR CONTRIBUTIONS}

Conceptualization: Jae-Moon Choi, Hong-Seuk Yang, Yong Beom Kim. Data curation: Hey-Ran Choi, Jae-Moon Choi, Hong-Seuk Yang, Junyong In, Yong Beom Kim. Formal analysis: Hey-Ran Choi, Hong-Seuk Yang, Junyong In, Yong Beom Kim. Funding acquisition: Hong-Seuk Yang. Methodology: Hey-Ran Choi, Jae-Moon Choi, Hong-Seuk Yang, Junyong In. Project administration: Hong-Seuk Yang. Writing original draft: Hey-Ran Choi, Chungon Park, Yong Beom Kim. Writing - review \& editing: Hey-Ran Choi, Jae-Moon Choi, Hong-Seuk Yang, Junyong In, Chungon Park, Yong Beom Kim. Investigation: Jae-Moon Choi, Hong-Seuk Yang, Junyong In, Chungon Park, Yong Beom Kim. Resources: JaeMoon Choi, Hong-Seuk Yang. Supervision: Hong-Seuk Yang.

\section{ORCID}

Hey-Ran Choi, https://orcid.org/0000-0002-9899-0158 Hong-Seuk Yang, https://orcid.org/0000-0003-2023-8705 Jae-Moon Cho, https://orcid.org/0000-0002-1161-6586 Chungon Park, https://orcid.org/0000-0002-8269-5058 Junyong In, https://orcid.org/0000-0001-7403-4287 Yong Beom Kim, https://orcid.org/0000-0003-2369-6525

\section{REFERENCES}

1. Beny K, Piriou V, Dussart C, Hénaine R, Aulagner G, Armoiry X; experts du NMBA pharmacoepidemiology group. [Impact of sugammadex on neuromuscular blocking agents use: a multicentric, pharmaco-epidemiologic study in French university hospitals and military hospitals]. Ann Fr Anesth Reanim 2013; 32: 838-43. French.

2. Pavoni V, Gianesello L, De Scisciolo G, Provvedi E, Horton D, Barbagli R, et al. Reversal of profound and "deep" residual rocuronium-induced neuromuscular blockade by sugammadex: a neurophysiological study. Minerva Anestesiol 2012; 78: 5429.

3. Woo T, Kim KS, Shim YH, Kim MK, Yoon SM, Lim YJ, et al. Sugammadex versus neostigmine reversal of moderate rocuronium-induced neuromuscular blockade in Korean patients. Korean J Anesthesiol 2013; 65: 501-7.

4. Bom A, Bradley M, Cameron K, Clark JK, Van Egmond J, Feilden $\mathrm{H}$, et al. A novel concept of reversing neuromuscular block: chemical encapsulation of rocuronium bromide by a cyclodextrin-based synthetic host. Angew Chem Int Ed Engl 2002; 41: 266-70.

5. Morris RB, Cronnelly R, Miller RD, Stanski DR, Fahey MR. Pharmacokinetics of edrophonium and neostigmine when antagonizing d-tubocurarine neuromuscular blockade in man. Anesthesiology 1981; 54: 399-401.

6. Donati F, McCarroll SM, Antzaka C, McCready D, Bevan DR. Dose-response curves for edrophonium, neostigmine, and pyridostigmine after pancuronium and d-tubocurarine. Anesthesiology 1987; 66: 471-6.

7. Caldwell JE. Clinical limitations of acetylcholinesterase antagonists. J Crit Care 2009; 24: 21-8.

8. Zwiers A, van den Heuvel M, Smeets J, Rutherford S. Assessment of the potential for displacement interactions with sugammadex: a pharmacokinetic-pharmacodynamic modelling approach. Clin Drug Investig 2011; 31: 101-11.

9. Choi H, Park SY, Kim YB, In J, Yang HS, Lee JS, et al. Effects of dexamethasone and hydrocortisone on rocuroniuminduced neuromuscular blockade and reversal by sugammadex in phrenic nerve-hemidiaphragm rat model. Korean J Anesthesiol 2019; 72: 366-74.

10. Choi JM, Kim HJ, Choi HR, Kim YB, Bae HJ, Yang HS. Remifentanil does not inhibit sugammadex reversal after rocuronium-induced neuromuscular block in the isolated hemidiaphragm of the rat: an ex vivo study. J Anesth 2019; 33: 642-6.

11. Kim YB, Yang HS, Kim HJ, Choi HR, In J, Yoon SY, et al. Effects of neuromuscular presynaptic muscarinic M1 receptor blockade on rocuronium-induced neuromuscular blockade in immobilized tibialis anterior muscles. Clin Exp Pharmacol Physiol 2018; 45: 1309-16.

12. Kim YB, Lee S, Choi HR, In J, Chang YJ, Kim HJ, et al. Effects of adenosine receptor agonist on the rocuroniuminduced neuromuscular block and sugammadex-induced recovery. Korean J Anesthesiol 2018; 71: 476-82.

13. Keegan MT. Endocrine pharmacology. editors. Pharmacology and physiology for anesthesia: foundations and clinical application. In: Hemmings HC, Egan TD, Philadelphia (PA), Elsevier. 2019. p. 708-31.

14. Liu MM, Reidy AB, Saatee S, Collard CD. Perioperative steroid management: approaches based on current evidence. Anesthesiology 2017; 127: 166-72.

15. Woodcock T, Barker P, Daniel S, Fletcher S, Wass JAH, Tomlinson JW, et al. Guidelines for the management of glucocorticoids during the peri-operative period for patients with adrenal insufficiency: guidelines from the Association of Anaesthetists, the Royal College of Physicians and the Society for Endo- 
crinology UK. Anaesthesia 2020; 75: 654-63.

16. Seo KH. Perioperative glucocorticoid management based on current evidence. Anesth Pain Med (Seoul) 2021; 16: 8-15.

17. Kam PJ, Heuvel MW, Grobara P, Zwiers A, Jadoul JL, Clerck Ed, et al. Flucloxacillin and diclofenac do not cause recurrence of neuromuscular blockade after reversal with sugammadex. Clin Drug Investig 2012; 32: 203-12.

18. Rezonja K, Sostaric M, Vidmar G, Mars T. Dexamethasone produces dose-dependent inhibition of sugammadex reversal in in vitro innervated primary human muscle cells. Anesth Analg 2014; 118: 755-63.

19. Gulec E, Biricik E, Turktan M, Hatipoglu Z, Unlugenc H. The effect of intravenous dexamethasone on sugammadex reversal time in children undergoing adenotonsillectomy. Anesth Analg 2016; 122: 1147-52

20. Chen W, Chang CE, Gilson MK. Calculation of cyclodextrin binding affinities: energy, entropy, and implications for drug design. Biophys J 2004; 87: 3035-49.
21. Chodera JD, Mobley DL. Entropy-enthalpy compensation: role and ramifications in biomolecular ligand recognition and design. Annu Rev Biophys 2013; 42: 121-42.

22. Fuchs-Buder T, Meistelman C, Raft J. Sugammadex: clinical development and practical use. Korean J Anesthesiol 2013; 65: 495-500.

23. Jones RK, Caldwell JE, Brull SJ, Soto RG. Reversal of profound rocuronium-induced blockade with sugammadex: a randomized comparison with neostigmine. Anesthesiology 2008; 109: 816-24.

24. Plaud B, Debaene B, Donati F, Marty J. Residual paralysis after emergence from anesthesia. Anesthesiology 2010; 112: 101322.

25. Kotake Y, Ochiai R, Suzuki T, Ogawa S, Takagi S, Ozaki M, et al. Reversal with sugammadex in the absence of monitoring did not preclude residual neuromuscular block. Anesth Analg $2013 ; 117: 345-51$ 